\title{
Developing Physicochemical Characterization and In vitro Release Test Methods to Probe Drug Release Mechanism from Multivesicular Liposomes
}

Soumyarwit Manna ${ }^{1,2}$, Yong $\mathrm{Wu}^{2}$, Peter Petrochenko ${ }^{1}$, Bonhye Koo ${ }^{1,2}$, Lynn Chen ${ }^{1,2}$, Yixuan Dong ${ }^{3}$, Xiaoming $\mathrm{Xu}^{3}$, Stephanie Choi ${ }^{1}$, Darby Kozak ${ }^{1}$, Yan Wang ${ }^{1}$, Jiwen Zheng ${ }^{2}$

${ }^{1}$ Division of Therapeutic Performance, Office of Research and Standards, Office of Generic Drugs, Center for Drug Evaluation and Research, Food and Drug Administration, Silver Spring, Maryland 20993, United States

2.Division of Biology, Chemistry and Materials Science, Office of Science and Engineering

Laboratories, Center for Devices and Radiological Health, Food and Drug Administration, Silver Spring, Maryland 20993, United States

3.Division of Product Quality Research, Office of Testing and Research, Office of Pharmaceutical Quality, Center for Drug Evaluation and Research, Food and Drug Administration, Silver Spring, Maryland 20993, United States

Multivesicular liposome (MVL), a lipid-based drug delivery system, was designed to provide sustainedrelease of drugs with short half-lives. Although many studies focused on encapsulating various drugs into MVLs, there is limited information on the mechanism of drug release from MVLs. Better understanding of the release mechanism(s) is useful to advancing MVL drug product development, quality assessment, and generic formulation equivalence. In the current study, EXPAREL ${ }^{\circledR}$ (bupivacaine (BPV) loaded MVL) was selected as the model product to study how the dissolution conditions of temperature and agitation speed affect the properties of drug release.

Particle size and morphology of the MVL's were measured using laser diffraction (LD), cryo-scanning electron microscopy (cryo-SEM) and confocal laser scanning microscopy (CLSM). The effect of temperature and agitation on the in vitro drug release testing (IVRT) was analyzed using a reverse dialysis set-up incorporated in a USP II apparatus. The dialysis tubes (MWCO: 100kDa) containing one $\mathrm{mL}$ of phosphate-buffered saline (PBS; pH 7) were suspended in $200 \mathrm{~mL}$ of PBS containing $\sim 0.53$ $\mathrm{mg} / \mathrm{mL}$ of BPV-MVLs. The concentration of BPV was detected real-time placing in-situ fiber optic UV inside the dialysis tubes $\left(\lambda_{\mathrm{BPV}}-263 \mathrm{~nm}\right)$. Studied IVRT conditions include: temperature $(25,31,37$ and $\left.40^{\circ} \mathrm{C}\right)$, agitation speed (120 and $\left.240 \mathrm{rpm}\right)$.

Prior to IVRT, cryo-SEM revealed the diameter (Mean \pm SD) of the spherical MVLs and characteristic inner "polyhedral" compartments were $23.6 \pm 11.5 \mu \mathrm{m}(\mathrm{N}=100)$ and $1.52 \pm 0.44 \mu \mathrm{m}(\mathrm{N}=500)$, respectively (Figs.1A-1C). Similar size was observed by LD (D50 - 25.5 $\pm 1.4 \mu \mathrm{m} ;(\mathrm{N}=12)$ ) and CLSM (Fig.1D). Post IVRT, cryo-SEM showed that the MVLs have undergone erosion, resulting in compromised morphology (Figs. 1E-1F). A tri-phasic release characteristics was revealed across all testing conditions, comprising of an initial burst phase, lag phase, and a secondary release phase. There was a minimal impact on the initial burst caused by temperature and agitation. However, the lag phase and the secondary release were significantly affected by temperature and agitation. The lag was reduced by increasing temperature $\left(25\right.$ to $\left.31^{\circ} \mathrm{C}\right)$ or agitation $(120$ to $240 \mathrm{rpm})$ (Fig. 2). However, the lag was prolonged when temperature further increased from 31 to $37^{\circ} \mathrm{C}$, indicating a possible reorganization of lipids. The lag was not observed at $40^{\circ} \mathrm{C}$, likely caused by continuous diffusion facilitated by the lipid phase transition $\left(\mathrm{T}_{\mathrm{m}} \sim 41^{\circ} \mathrm{C}\right)$. 
The findings from the current study enables improved understanding of the morphology and drug release mechanism from MVLs. When optimized, such physicochemical characterization and IVRT methods can be used to ensure product quality or to support the equivalence between the reference listed drug and a generic MVL product.

\section{References}

[1] P Chahar, K Cumminngs III, J. Pain Res 5 (2012), p. 257-264

[2] S Mantripragada, Prog Lipid Res, 41(5) (2002), p. 392-406

[3] This project was supported in part by an appointment to the Research Participation Program at Center for Drug Evaluation and Research administered by the Oak Ridge Institute for Science and Education through an agreement between the U.S. Department of Energy and FDA. The authors would like to acknowledge FDA Advanced Characterization Facility (ACF), CDRH/OSEL/DBCMS and CDER/OPQ/OTR for instrument use. Soumyarwit Manna, Yong Wu, Bonhye Koo, Lynn Chen and Yixuan Dong were supported in part by an appointment to the Research Participation Program at the U.S. Food and Drug Administration (FDA), administered by the Oak Ridge Institute for Science and Education through an interagency agreement between the U.S. Department of Energy and the FDA.

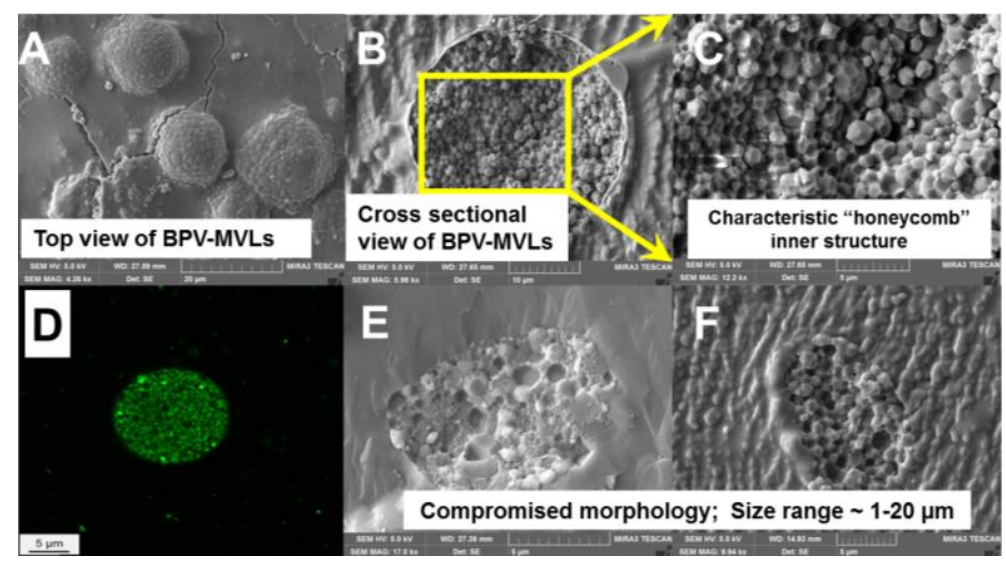

Figure 1. Prior to IVRT, Cryo-SEM images showing A) Top view; B) Cross-sectional view; C) Inner structure and CLSM image showing D) Cross-sectional view of the BPV-MVLs. Post IVRT, Cryo-SEM images showing disintegration of the BPV-MVLs and inner-structure (E-F).

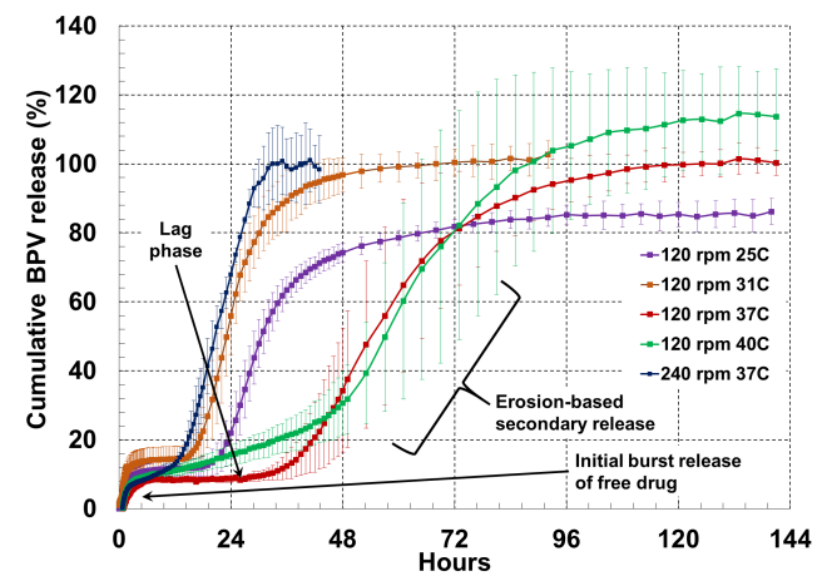

Figure 2. BPV release profiles (Mean $\pm \mathrm{SD})$ at different agitation speeds and temperatures $(\mathrm{N}=3$ for each condition) 